\title{
Internal waves and synchronized precession in a cold vapor
}

\author{
M. Ö. Oktel ${ }^{a}$ and L. S. Levitov ${ }^{b}$ \\ ${ }^{a}$ Department of Physics, Ohio State University, 174 West 18 Avenue, Columbus, OH 43210 \\ ${ }^{b}$ Department of Physics, Massachusetts Institute of Technology, 77 Massachusetts Ave, Cambridge, MA 02139
}

(October 29, 2018)

\begin{abstract}
Exchange in a Boltzmann gas of bosons with several internal states leads to collective transport of internal polarization. The internal dynamics can be understood as Larmor precession in the presence of a torque induced by atoms on each other via exchange coupling. A generalized Bloch equation that includes interatomic exchange effects as well as orbital motion in the gas is derived and used to interpret recent experiment by Lewandowski et al. [1] as an excitation of a collective wave of internal state polarization. It is shown that exchange leads to formation of domains in which precession frequencies are synchronized.
\end{abstract}

Atomic gases in the cold collision regime characterized by de Broglie wavelength long compared to the range of interparticle potential represent an interesting quantum many-body system. Most surprisingly, spin waves in a cold spin polarized gas are collective excitations. This phenomenon was actively studied in the 80 's, first predicted by Bashkin [2] and independently by Lhuillier and Laloë [3], and confirmed by NMR experiments in spin polarized $H \downarrow$ by Johnson et al. 迎, in ${ }^{3} \mathrm{He}$ by Nacher et al. [5], and in dilute ${ }^{3} \mathrm{He}-{ }^{4} \mathrm{He}$ mixtures by Gully and Mullin [6]. A detailed quantitative theory of the observed NMR spectra was given by Levy and Ruckenstein [7]. Bigelow et al. [8] demonstrated that collective spin waves are preserved even in the Knudsen regime. The theory was further developed by Miyake et al. [9] and reviewed in [10].

Exchange effects in gases are not limited to spin phenomena, since any pair of internal states can play a role similar to spin states in exchange collisions [11]. Apart from new energy scales arising due to internal states spectrum, the main difference is in the anisotropic character of exchange. For generic internal states the Hamiltonian does not have spin-rotational symmetry, which opens a number of new interesting possibilities. Verhaar et al. [13] demonstrated that interatomic exchange leads to enhancement (by a factor of two) of the density shift of Rabi transition. Tiesinga et al. 14. and Kokkelmans et al. 15 considered application of this effect in Cesium fountain clock. Similar exchange enhancement occurs in the optical spectrum density shift. The theory [2,3] of collective spin-waves, can be straightforwardly adapted to describe optical excitations [12].

New aspects of cold collision exchange arise in experiments on Bose-Einstein condensation (BEC) in trapped gases. The exchange part of the density shift is absent in $\mathrm{BEC}$ at $T=0$ and is reduced at $0<T<T_{\mathrm{BEC}}$ [12, 16, 17]. Interestingly, in this case all modes involving coupling of internal states are split into doublets [18].

In a recent experiment [1] Rabi transition was studied in a cigar shaped sample of $\mathrm{Rb}$ vapor contained in IoffePritchard trap. The $\left|F, m_{f}\right\rangle=|1,-1\rangle,|2,1\rangle$ levels of the hyperfine multiplet of Rb split by $\omega_{0} \approx 6.8 \mathrm{GHz}$ were used. Almost perfect compensation of the density shift by spatially varying Zeeman frequency was achieved. The transition frequency varied along the sample axis by few tens of $\mathrm{Hz}$. Initial inner state with polarization in the $x-y$ plane was prepared by a $\frac{\pi}{2}$ pulse. It was observed that the polarization does not remain in the $x-y$ plane during free Larmor precession. This was argued to result from spatial segregation of atoms with different $z$ spin components. However, the confinement potential [1] spin dependence was very weak, and the best estimate of segregation due to the difference in mechanical forces experienced by atoms with different spins was at least an order of magnitude longer than the time $\approx 0.2 \mathrm{~s}$ of the $z$ component buildup.

We argue below that the phenomena of Ref. [1] are explained by coherent evolution of atoms' internal state rather than by mechanical segregation in the gas. The observed $z$ component profile is readily accounted for by interatomic exchange coupling. The transition frequency [1] varies along the sample axis, and a short time after precession started a gradient of precession angle builds up. Now, consider two interacting atoms with slightly different polarization due to spatially varying Larmor frequency. The exchange interaction of these atoms can be described [11] as precession of each atom's spin around the net spin of both atoms. Since both atoms have transverse polarization, the precession about a net spin (which is also transverse) will move the spins out of the $x-y$ plane and both of them will acquire a finite $z$ component.

Exchange effects can be illustrated by a thought experiment involving a gas of identical atoms with density $n$ and spin $1 / 2$ contained in a box. Take the spin polarization $\mathbf{s}$ to be purely transverse and the same for all atoms. For isotropic exchange coupling $\mathcal{H}=$ $\hbar \int\left(\omega_{0} s^{z}(r)+\frac{\lambda}{2} \mathbf{s}(r) \cdot \mathbf{s}(r)\right) d^{3} r$ the polarization $\mathbf{s}$ is uniformly precessing, $\mathbf{s}(t)=\frac{1}{2} n\left(\cos \omega_{0} t \hat{\mathbf{x}}+\sin \omega_{0} t \hat{\mathbf{y}}\right)$. Now consider a test atom passing through the box with spin polarization different from that of the other atoms. The test atom spin will experience an effective 'magnetic' field $\mathbf{B}=\omega_{0} \hat{\mathbf{z}}+\lambda \mathbf{s}(t)$ with the exchange part $\lambda \mathbf{s}(t)$ giving rise to Rabi transitions. In a Larmor coordinate frame rotating with frequency $\omega_{0}$ about the $z$ axis the effective field 
is just $\lambda n$ along the gas polarization $\mathbf{s}$, time-independent in this frame. Since $\mathbf{s}$ is transverse, Rabi transition will generate a $z$ component of the test atom polarization, even if initially it was in the $x-y$ plane.

Before accepting this explanation one needs to discuss energy conservation. The probabilities to find the test atom in the up and down states after coming out of the box differ from those in the initial state, since its $z$ spin component changes. This means that the test atom energy can change by $\hbar \omega_{0}$. The total energy of the system, however, does not change because the spins coupled by exchange precess together around the net spin so that the total spin is conserved [11]. The change of the net spin $z$ component in the box is equal and opposite to the test atom spin change, as required by the energy balance.

Although everything is consistent with energy conservation, the energy change of $\hbar \omega_{0}$ with $\omega_{0} \approx 6.8 \mathrm{GHz}$ much higher than other frequencies in the system [1] may appear counter-intuitive. The temperature $T=600 \mathrm{nK}[1]$ corresponds to $k_{B} T / \hbar \sim 10 \mathrm{KHz}$, the trap frequencies are $\left(\omega_{\perp}, \omega_{z}\right)=(230,7) \mathrm{Hz}$. However, the characteristic exchange frequency is $\lambda n \simeq 140 \mathrm{~Hz}$ for the typical density $n=210^{13} \mathrm{~cm}^{-3}$ (see below). This is much higher than the transition frequency broadening estimated from the precession decay time to be of order of few $\mathrm{Hz}$ [1]. This makes the exchange induced Rabi transitions at the energy $\hbar \omega_{0}$ fully coherent, despite that $\hbar \omega_{0} \gg \lambda n$.

The length corresponding to one Rabi cycle is

$$
l_{\mathrm{exch}}=\frac{v_{\mathrm{T}}}{\lambda n}=16 \mu \mathrm{m}, \quad\left(v_{\mathrm{T}}=\sqrt{2 T / m}\right)
$$

This is larger than the sample radius $r_{\perp}=7.3 \mu \mathrm{m}$ but much smaller than the sample length $r_{z}=240 \mu \mathrm{m}$. Since movement of an atom by $\simeq \frac{1}{4} l_{\text {exch }}$ is sufficient for rotating the spin by $\frac{\pi}{2}$ and moving it out of the $x-y$ plane, the exchange coupling is a viable mechanism for spin reorientation in this system.

The separation of $\mathrm{Rb}$ atoms into a gas sample and a test particle in the thought experiment is artificial. The atoms in [1] share both roles, by inducing precession on each other via exchange coupling. One therefore has to consider collective dynamics of atom polarization [2,3]. The Hamiltonian of $\mathrm{Rb}$ atoms in a trap has the form

$$
\begin{aligned}
& \mathcal{H}=\int\left(\sum_{j=1,2} \bar{\psi}_{j} H_{j} \psi_{j}+\frac{\hbar}{2} \sum_{j, k=1,2} \lambda_{j k}: \hat{n}_{j} \hat{n}_{k}:\right) d^{3} r \\
& H_{j}=-\frac{\hbar^{2}}{2 m} \nabla^{2}+U_{j}(r), \quad \lambda_{j k}=\frac{4 \pi \hbar}{m} a_{j k}
\end{aligned}
$$

where $\hat{n}_{j}=\bar{\psi}_{j} \psi_{j}$ is the density operator. For the states used in Ref. [1] the scattering lengths are $\left(a_{11}, a_{22}, a_{12}\right)=$ $(100.9,95.6,98.2) a_{0}$ with $a_{0}$ the Bohr's radius.

The polarization of internal states is described by 'spin' operators with components given by Pauli matrices

$$
\hat{s}^{x(y, z)}(r)=\frac{1}{2} \sum_{j, k} \bar{\psi}_{j}(r) \sigma_{j k}^{x(y, z)} \psi_{k}(r)
$$

and standard commutation algebra

$$
\left[\hat{s}^{\alpha}(r), \hat{s}^{\beta}\left(r^{\prime}\right)\right]=i \varepsilon_{\alpha \beta \gamma} \hat{s}^{\gamma}(r) \delta\left(r-r^{\prime}\right)
$$

of spin density operators.

The system [1] is deep in the cold collision regime, since thermal de Broglie wavelength $\lambda_{\mathrm{T}}=h / m v_{\mathrm{T}} \simeq 4000 a_{0}$ is much larger than the scattering lengths $a_{j k}$. We employ the forward scattering approximation also known as the random phase approximation [19]. The interaction can be rewritten in momentum representation as

$$
\begin{aligned}
& \int: \hat{n}_{j} \hat{n}_{k}: d^{3} r=\sum_{p+p^{\prime}=p^{\prime \prime}+p^{\prime \prime \prime}} \bar{\psi}_{j, p} \bar{\psi}_{k, p^{\prime}} \psi_{j, p^{\prime \prime}} \psi_{k, p^{\prime \prime \prime}} \\
& =\sum_{p, p^{\prime}, q}(6) \\
& \left(\bar{\psi}_{j, p_{+}} \bar{\psi}_{k, p_{-}^{\prime}} \psi_{j,-p_{-}} \psi_{k,-p_{+}^{\prime}}+\bar{\psi}_{j, p_{+}} \bar{\psi}_{k, p_{-}^{\prime}} \psi_{j,-p_{+}^{\prime}} \psi_{k,-p_{-}}\right)
\end{aligned}
$$

where $p_{ \pm}=p \pm q / 2$. The first term of (6) accounts for the forward scattering process, while the second term describes exchange scattering. Identifying the operators in (6) with the spin density components (4) we obtain

$$
\begin{gathered}
: \hat{n}_{1} \hat{n}_{1}:=\frac{1}{2}\left(\hat{n}+2 \hat{s}^{z}\right)^{2}, \quad: \hat{n}_{2} \hat{n}_{2}:=\frac{1}{2}\left(\hat{n}-2 \hat{s}^{z}\right)^{2} \\
: \hat{n}_{1} \hat{n}_{2}+\hat{n}_{2} \hat{n}_{1}:=\frac{1}{2} \hat{n}^{2}-2\left(\hat{s}^{z}\right)^{2}+4 \hat{s}^{+} \hat{s}^{-}+4 \hat{s}^{-} \hat{s}^{+}
\end{gathered}
$$

In the spin representation the interaction has the form

$$
\frac{1}{2} \sum_{j, k} \lambda_{j k}: \hat{n}_{j} \hat{n}_{k}:=\frac{u}{2} \hat{n}^{2}+\Lambda \hat{n} \hat{s}^{z}+\delta \lambda\left(\hat{s}^{z}\right)^{2}+\lambda_{12} \mathbf{s}^{2}
$$

with $u=\lambda_{11}+\lambda_{22}+\lambda_{12}, \Lambda=\lambda_{11}-\lambda_{22}$, and $\delta \lambda=$ $\lambda_{11}+\lambda_{22}-2 \lambda_{12}$. Spin dynamics is given by $\partial_{t} \hat{\mathbf{s}}=\frac{i}{\hbar}[\hat{\mathbf{s}}, \mathcal{H}]$, where the commutator can be evaluated with the help of the relations (5). After taking the expectation values $\mathbf{s}=\langle\hat{\mathbf{s}}\rangle$ we obtain a generalized Bloch equation

$$
\partial_{t} \mathbf{s}+\vec{\nabla} \cdot \overrightarrow{\mathbf{j}}=\boldsymbol{\Omega} \times \mathbf{s}, \quad \boldsymbol{\Omega}=\left(\omega_{0}+\delta \omega\right) \hat{\mathbf{z}}+2 \lambda_{12} \mathbf{s}
$$

with $\overrightarrow{\mathbf{j}}(r)=-\frac{i \hbar}{2 m}\left\langle\bar{\psi}_{j} \mathbf{s}_{j k} \vec{\nabla} \psi_{k}\right\rangle+$ h.c. the spin current. Here

$$
\delta \omega(r)=\frac{1}{\hbar}\left(U_{1}-U_{2}\right)+\Lambda n+2 \delta \lambda s^{z}
$$

To make contact with the discussion in Ref. [1] we note that $\frac{1}{2} n \pm s^{z}=n_{1(2)}$, the occupation probabilities for the up and down spin. Combined with the form of $\Lambda$ and $\delta \lambda$, the frequency $\delta \omega(r)$ can be rewritten as

$$
\delta \omega(r)=\frac{1}{\hbar}\left(U_{1}-U_{2}\right)+2\left(\lambda_{11}-\lambda_{12}\right) n_{1}-2\left(\lambda_{22}-\lambda_{12}\right) n_{2}
$$

The first term is the Zeeman frequency shift due to the trap field inhomogeneity, while the last two terms (identical to Eq.(1) of Ref. [1]) give the density shift. The term $2 \lambda_{12} \mathrm{~s}$ in the expression (10) for $\boldsymbol{\Omega}(r)$ representing the effect of exchange is not considered in Ref. [1]. The role of this term is subtle. It drops out from the Bloch equation (10) for $\mathbf{s}$, since $\mathbf{s} \times \mathbf{s}=0$. However, since typically $2 \lambda_{12}|\mathbf{s}| \gg|\delta \omega(r)|$, this term should be taken into account in the Bloch equation for other spin-related quantities, 
such as the spin current $\overrightarrow{\mathbf{j}}$. The torque $\mathbf{s} \times \mathbf{j}$ (which in general is not along the $z$ axis) makes the spin current precess so that the $x, y$ and $z$ components intermix.

The transport equation for the spin current $\overrightarrow{\mathbf{j}}$ is derived in a similar fashion [2, 3]. We obtain

$$
\partial_{t} \overrightarrow{\mathbf{j}}+\alpha \vec{\nabla} \mathbf{s}=\boldsymbol{\Omega}(r) \times \overrightarrow{\mathbf{j}}-\gamma \overrightarrow{\mathbf{j}}, \quad \alpha=\frac{1}{3} v_{\mathrm{T}}^{2}
$$

where the elastic collision rate $\gamma=4 \pi a^{2} v_{\mathrm{T}} n \simeq 20 \mathrm{~Hz}$ with parameters of Ref. [1]. The term $\alpha \nabla \mathbf{s}$ arises in a standard way after retaining angular harmonics of the lowest order in the transport equation. In Eq.(13) we ignored the terms such as $\mathbf{s} \nabla\left(U_{1}+U_{2}\right)$ and $n \hat{\mathbf{z}} \nabla\left(U_{1}-U_{2}\right)$, since their magnitude is small (see Ref. [1]). In this approximation, the spin and density dynamics decouple, in agreement with the observation [1].

Since the interaction parameters $\lambda_{j k}$ for $\mathrm{Rb}$ coincide within $3 \%$, spin is approximately conserved by elastic collisions. In this case, the spin current relaxation rate $\gamma$ is the same as for the average particle momentum, and no spin relaxation appears in the Bloch equation (10). Elastic collisions control spin relaxation indirectly, by making spin diffusion constant complex [2,3].

The transport equations (10), (13) can be simplified for a one dimensional system [1] by averaging over sample crossection. Large exchange $\lambda n \simeq \omega_{\perp}$ [1] leads to fast dynamical averaging of spin polarization in each crossection with parameters slowly varying along the sample length. In averaging Eqs. (10),(13) we assume Gaussian density profile $n(\rho)=n e^{-\rho^{2} / r_{\perp}}$. The averaging of the terms in Eqs.(10),(13) quadratic in density and/or spin is performed as $\int n^{2}(\rho) d^{2} \rho / \int n(\rho) d^{2} \rho=\frac{1}{2} n$, where $n$ is the peak density. After rescaling all coupling constants

$$
\lambda_{j k} \rightarrow \frac{1}{2} \lambda_{j k}
$$

and replacing $\nabla$ by one dimensional $\partial_{x}$ we obtain transport equations of Leggett-Rice form [20]

$$
\begin{aligned}
& \partial_{t} \mathbf{s}+\partial_{x} \mathbf{j}=\left(\omega_{0}+\widetilde{\delta \omega}(x)\right) \hat{\mathbf{z}} \times \mathbf{s} \\
& \partial_{t} \mathbf{j}+a \partial_{x} \mathbf{s}=\left(\left(\omega_{0}+\widetilde{\delta \omega}(x)\right) \hat{\mathbf{z}}+\lambda_{12} \mathbf{s}\right) \times \mathbf{j}-\gamma \mathbf{j} \\
& \widetilde{\delta \omega}(x)=\frac{1}{\hbar}\left(U_{1}-U_{2}\right)+\frac{1}{2} \Lambda n+\delta \lambda s^{z}
\end{aligned}
$$

The coupled dynamics of $\mathbf{s}$ and $\mathbf{j}$ is nonlinear because of the exchange precession torque $\lambda_{12} \mathbf{s} \times \mathbf{j}$ in Eq.(16).

In the approximation $\widetilde{\delta \omega}(x), \gamma \ll \lambda_{12} n$ [1] one can simplify transport equations by performing a gradient expansion. We first go to the Larmor frame rotating with frequency $\omega_{0}$, which eliminates $\omega_{0}$ from Eqs.(15),(16). Next, ignoring the time derivative $\partial_{t} \mathbf{j}$ in Eq. 16 we solve it for $\mathbf{j}$ in terms of $\mathbf{s}$ and $\partial_{x} \mathbf{s}$, and substitute the result in Eq.15). This gives the Landau-Lifshitz equation [21]

$$
\begin{gathered}
\partial_{t} \mathbf{s}-\partial_{x}\left(D_{1}(\mathbf{s}) \partial_{x} \mathbf{s}\right)=\left[\widetilde{\delta \omega}(x) \hat{\mathbf{z}}-D_{2}(\mathbf{s}) \partial_{x}^{2} \mathbf{s}\right] \times \mathbf{s} \\
D_{1}(\mathbf{s})=\frac{\alpha \gamma}{\gamma^{2}+\lambda_{12}^{2} \mathbf{s}^{2}}, \quad D_{2}(\mathbf{s})=\frac{\alpha \lambda_{12}}{\gamma^{2}+\lambda_{12}^{2} \mathbf{s}^{2}}
\end{gathered}
$$

It is convenient to nondimensionalize Eq.(18). We rescale $\mathbf{s}$ by $|\mathbf{s}|_{\max }=\frac{1}{2} n$, the frequency $\widetilde{\delta \omega}(x)$ and scattering rate $\gamma$ by $\lambda_{12}|\mathbf{s}|_{\max }$, and choose as length unit

$$
\frac{\lambda_{12}|\mathbf{s}|_{\max }}{\alpha^{1 / 2}} \equiv \frac{\lambda_{12} n}{2}\left(\frac{3 m}{2 k_{\mathrm{B}} T}\right)^{1 / 2} \equiv \frac{\sqrt{3}}{2} l_{\mathrm{exch}} \approx 14 \mu \mathrm{m}
$$

Eq.(18) preserves its form, with $D_{2}=1 /\left(\gamma^{2}+\mathbf{s}^{2}\right)$ and $D_{1}=\gamma D_{2}$. The dimensionless damping $\gamma$ is

$$
\gamma=\frac{4 \pi a^{2} v_{\mathrm{T}} n}{\frac{1}{2} \lambda_{12} n}=\frac{2}{\hbar} a m v_{\mathrm{T}}=4 \pi \frac{a}{\lambda_{\mathrm{T}}} \simeq 0.31
$$

since $a \approx 100 a_{0}$ and the de Broglie wavelength $\lambda_{\mathrm{T}}=$ $h / m v_{\mathrm{T}} \approx 4000 a_{0}$ [1].

The results of numerical simulation of Eq.(18) are shown in Fig.1. The spatial and temporal behavior is similar to that in Ref. [1]: The $z$ component builds up $\simeq 0.2 \mathrm{~s}$ after precession started and then gradually decays to zero along with the oscillating transverse component.
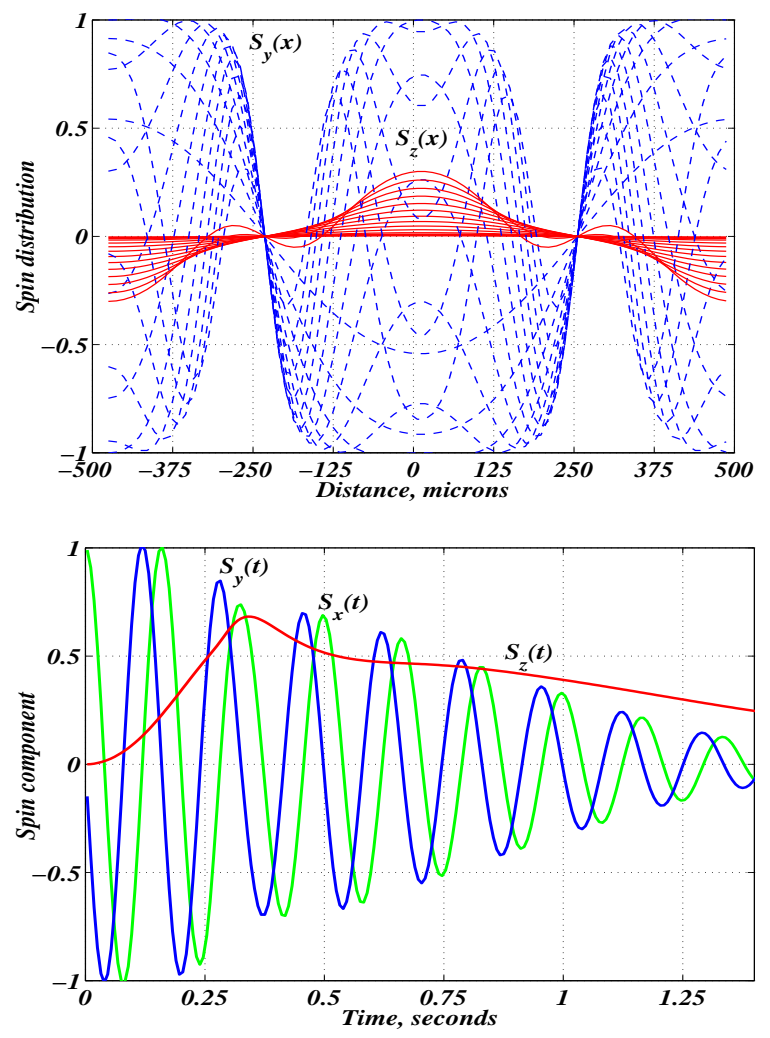

FIG. 1. Numerical simulation of Eq.(18). Parameters used: constant density $n=210^{13} \mathrm{~cm}^{-3}$, exchange frequency $\lambda_{12}|\mathbf{s}|_{\max }=70 \mathrm{~Hz}$. Spatial variation of the transition frequency $\widetilde{\delta \omega}(x)=-\Omega \cos (2 \pi x / L)$ with $\Omega=20 / \pi \approx 6.37 \mathrm{~Hz}$ and sample size $L=10^{3} \mu \mathrm{m}$. Top: spin distribution evolution at $0<t<0.2 \mathrm{sec}$; Bottom: time dependence at $x=0$. 
Spin precession becomes synchronized in different parts of the sample (see Fig.2), due to compensation of the transition frequency $\widehat{\delta \omega}(x)$ spatial variation by the exchange field $\lambda_{12} \mathbf{s}(x)$. In our simulation, synchronization takes place independently in the domains with $s_{z}>0$ and $s_{z}<0$. Frequency was evaluated as

$$
f=\frac{1}{2 \pi} d \theta / d t, \quad \theta=\arg \left(s_{x}+i s_{y}\right)
$$

During the first $0.2 \mathrm{~s}$ of the $z$ component buildup the frequency evolves from initial value $f=\frac{1}{2 \pi} \widetilde{\delta \omega}(x)$ to a constant value $\approx \pm 6 \mathrm{~Hz}$ in each domain.

While precession frequencies become synchronized, the phase $\theta$ varies within each domain producing spin flux between the domains. Spin density $\mathbf{s}(x)$ vanishes at the domain boundaries $x= \pm \frac{1}{4} L= \pm 250 \mu \mathrm{m}$ (see Figs.12,2). The number of synchronized domains and domain-specific frequency values in general depend on the amplitude and characteristic spatial scale of $\widetilde{\delta \omega}(x)$.
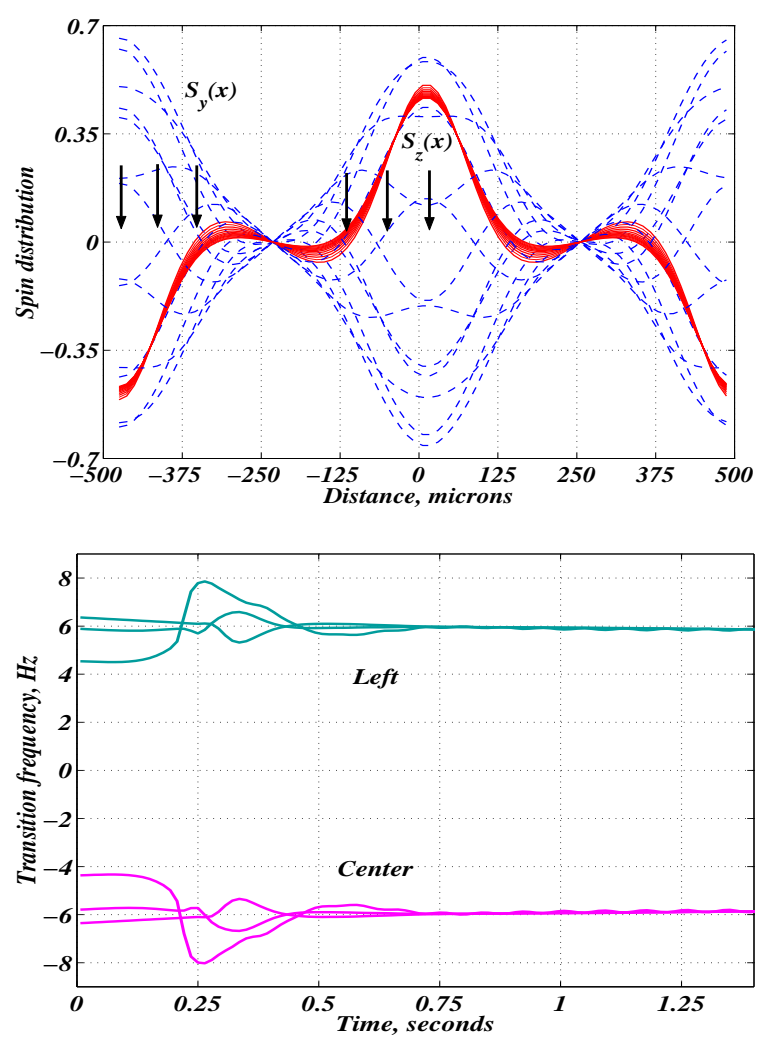

FIG. 2. Top: spin distribution evolution from $0.5 \mathrm{sec}$ to $0.7 \mathrm{sec}$ (with the same parameters as in Fig.11); Bottom: frequency (22) synchronization at the points marked by arrows.

The mechanism of transverse spin component decay in the synchronized state is polarization mixing caused by spin current between different domains. The time scale of spin decay, set by spin diffusion, is much longer than the elastic collision time. The $z$ component first builds up due to spin currents and then decays due to (longitudinal) diffusion, with characteristic time $(L / 2 \pi)^{2} \gamma / \alpha \approx$ 0.5 secs (see Fig[1). This is consistent with Ref. [1].
In summary, exchange coupling in a trapped gas leads to complex collective dynamics of polarization. Polarized atoms exert torque on the spin current creating a $z$ component profile in the presence of spatially varying transition frequency. Results of numerical simulation of the dynamics of the Rb system [1] are in agreement with observations. Surprisingly, the buildup of the $z$ component is accompanied by synchronization of precession frequencies. In the inhomogeneous state the sample breaks into two or more synchronized domains. Spin relaxation is caused by spin currents between the domains.

Synchronized precession should manifest itself in experiment as transition frequency locking to one value in the entire sample, if it is a single domain. Several synchronized domains formed within the sample will give rise to several plateaus in the transition frequency spatial dependence. Spin density vanishing between different domains should be observable by the spatially resolved Ramsey fringes technique of Ref. [1]

We are grateful to E. A. Cornell and Tin-Lun Ho for useful discussions.

[1] H. J. Lewandowski, D. M. Harber, D. L. Whitaker and E. A. Cornell, "Observation of anomalous spin-state segregation in a trapped ultra-cold vapor," cond-mat/0109476

[2] E. P. Bashkin, Pis'ma Zh. Eksp. Teor. Fiz. 33, 11 (1981) [JETP Lett. 33, 8 (1981)]; Zh. Eksp. Teor. Fiz. 87, 1948 (1984) [Sov. Phys. JETP 60, 1122 (1985)]

[3] C. Lhuillier and F. Laloë, J. Physique 43, 197 (1982); J. Physique 43, 225 (1982)

[4] B. R. Johnson, et al. Phys. Rev. Lett. 52, 1508 (1984)

[5] P. J. Nacher, G. Tastevin, M. Luduc, F. Laloë, J. Phys. (Paris) Lett. 45, L411 (1984);

G. Tastevin, P. J. Nacher, M. Luduc, F. Laloë, J. Phys. (Paris) Lett. 46, 249 (1985)

[6] W. J. Gully and W. J. Mullin, Phys. Rev. Lett. 52, 1810 (1984)

[7] L. P. Lévy and A. E. Ruckenstein, Phys. Rev. Lett. 52, 1512 (1984)

[8] N. P. Bigelow, J. H. Freed, and D. M. Lee, Phys. Rev. Lett. 63, 1609 (1989)

[9] K. Miyake, W. Mullin, and P. C. E. Stamp, J. Physique 46, 663 (1985)

[10] E. P. Bashkin, Usp. Fiz. Nauk 148, 433 (1986) [Sov. Phys. Usp. 29, 238 (1986)]

[11] B. J. Verhaar, in: Proceedings of the 14-th International Conference on Atomic Physics, eds. D. J. Wienland, C. E. Wieman, S. J. Smith, p.351 (AIP Press, 1995)

[12] M. Ö. Oktel, L. S. Levitov, Phys. Rev. Lett. 83, 6 (1999)

[13] B. J. Verhaar, J. M. V. A. Koelman, H. T. C. Stoof, O. J. Luiten, Phys. Rev. A35, 3825 (1987)

[14] E. Tiesinga, B. J. Verhaar, H. T. C. Stoof, D. van Bragt, Phys. Rev. A45, 2671 (1992) 
[15] S. J. J. M. F. Kokkelmans, B. J. Verhaar, K. Gibble and D. J. Heinzen, Phys. Rev. A 56, R4389 (1997)

[16] M. Ö. Oktel, T. C. Killian, L. S. Levitov, D. Kleppner, "Cold Collision Frequency Shift of an Optical Spectrum of a Trapped Gas," condmat/9911020

[17] C. J. Pethick, H. T. C. Stoof, Phys. Rev. A 6401 (1): 3618 (2001)

[18] M. Ö. Oktel, L. S. Levitov, "Collective dynamics of in- ternal states in a Bose gas," condmat/0108052

[19] A. A. Abrikosov, L. P. Gorkov, and I. E. Dzyaloshinskii, Methods of Quantum Field Theory in Statistical Physics (Dover, New York, 1975), Chap. 5

[20] A. J. Leggett, M. J. Rice, Phys. Rev. Lett. 20, 586 (1968)

[21] L. D. Landau, E. M. Lifshitz, Phys. Z. d. Sowjetunion, 8, $153(1935)$ 